\title{
A Plasmonic Refractive-Index Sensor Based Multiple Fano Resonance Multiplexing in Slot-Cavity Resonant System
}

\author{
Zicong GUO ${ }^{*}$, Kunhua WEN ${ }^{1}$, Yuwen QIN ${ }^{2}$, Yihong FANG ${ }^{1}$, \\ Zhengfeng $\mathrm{LI}^{1}$, and Li CHEN ${ }^{1}$
}

\author{
${ }^{1}$ School of Physics and Optoelectronic Engineering, Guangdong University of Technology, Guangzhou 510006, China \\ ${ }^{2}$ School of Information Engineering, Guangdong University of Technology, Guangzhou 510006, China \\ *Corresponding author: Zicong GUO_E-mail: 2111915043@mail2.gdut.edu.cn
}

\begin{abstract}
In this paper, a sub-wavelength metal-insulator-metal (MIM) waveguide structure is proposed by using a cross-shape rectangular cavity, of which wings are coupled with two rectangular cavities. Firstly, a cross-shape rectangular cavity is placed between the input and output MIM waveguides. According to the mutual interference between bright and dark modes, three Fano resonant peaks are generated. Secondly, by adding a rectangular cavity on the left wing of the cross shaped one, five asymmetric Fano resonance peaks are obtained. Thirdly, six asymmetric Fano resonance peaks are achieved after adding another cavity on the right wing. Finally, the finite-difference-time-domain (FDTD) method and multimode interference coupled-mode theory (MICMT) are used to simulate and analyze the coupled plasmonic resonant system, respectively. The highest sensitivity of $1000 \mathrm{~nm} / \mathrm{RIU}$ is achieved.
\end{abstract}

Keywords: Sub-wavelength; MIM waveguide; Fano resonance

Citation: Zicong GUO, Kunhua WEN, Yuwen QIN, Yihong FANG, Zhengfeng LI, and Li CHEN, "A Plasmonic Refractive-Index Sensor Based Multiple Fano Resonance Multiplexing in Slot-Cavity Resonant System,” Photonic Sensors, 2022, 12(2): $175-184$.

\section{Introduction}

Firstly, Fano resonance was proved to be caused by coherent interference between discrete state and continuous state in the atomic system [1-3]. Secondly, Fano resonance has the characteristics of high refractive index sensitivity, high preferred value, and strong field enhancement, showing ultra-clear and asymmetric lines. Therefore, the unique characteristics make Fano resonance applicable to various optical fields, such as filters, splitters, and sensors [4-10]. In particular, Fano resonances recently have been observed in the subwavelength metal-insulator-metal (MIM) waveguide structures. Because the plasmonic MIM waveguide is one of the most promising ways to obtain high integrated photonic circuit, Fano resonances in MIM waveguide structures have attracted many researchers' attention [11-17]. In recent years, many different MIM waveguide structures have been designed and asymmetric Fano peak can be obtained in the visible to near infrared wavelength range [18-26]. For considering the development of integrated photonics and the parallel processing capacity with multiple Fano channels, people begin to explore the possibility of generating more Fano resonance peaks by designing compact MIM structures [27, 28].

\footnotetext{
Received: 4 November 2020 / Revised: 1 March 2021

(C) The Author(s) 2021. This article is published with open access at Springerlink.com DOI: $10.1007 / \mathrm{s} 13320-021-0631-8$
}

Article type: Regular 
In this paper, three Fano resonances are firstly realized in a cross-shape MIM waveguide structure owing to the mutual interference between the bright and dark resonant modes. Then, two rectangular cavities are successively added at both ends of the cross-shape one to obtain more effects of mode interactions. Consequently, up to six asymmetrical Fano resonant peaks are generated in this proposed structure. The performance of the MIM structure is analyzed by the finite-difference-time-domain (FDTD) method and multimode interference coupled-mode theory (MICMT), respectively.

\section{Structure and discussion}

In Fig. 1(a), the horizontal and vertical parts of the cross-shape cavity resonator are considered as a classical end-coupled Fabry-Pero (FP) resonator, respectively. They can respectively provide the bright and dark resonant modes, of which the interaction can induce Fano resonance. The widths of the MIM waveguide and the rectangular resonator are represented by $D$. The distances between the cross-shape cavity and the input/output waveguide are denoted by $g$, and the lengths of vertical rectangular cavity and horizontal one are defined by $L$ and $S$, respectively. During the FDTD simulations, the following parameters remain the same all through the paper: the width is $D=50 \mathrm{~nm}$, the length is $L=640 \mathrm{~nm}$, the lengths of the upper and below parts of the vertical cavity are $l_{1}=410 \mathrm{~nm}$ and $l_{2}=$ $180 \mathrm{~nm}$, respectively, and the lengths of both wings of the horizontal cavity are the same as $S=190 \mathrm{~nm}$. Gap $(g)$ of $10 \mathrm{~nm}$ requires challenging thickness control of metal, but some teams have solved this problem. Wei [29] used the oxygen plasma etching technology to reduce the spacing of metal triangular nanoparticles in the MIM structure to $10 \mathrm{~nm}$ scale. Miyazaki et al. [30] considered a dielectric with a thickness of $T$ between two noble metal plates in a MIM waveguide. For $T$ values less than $10 \mathrm{~nm}$, small wavelength plasmons with wavelength of $10 \mathrm{~nm}$ can be obtained from visible light to the near infrared. In this paper, deposition of nanometer-thick thin film is sufficiently feasible with conventional techniques. Therefore, the distance $g$ is defined as $10 \mathrm{~nm}$. A uniform set of perfectly matched layers is used in the structure as absorbing boundary conditions. Firstly, we define the metal and dielectric materials as silver and air, respectively, and obtain the dielectric constant from the experimental data [31]. Specifically, there are two kinds of modes with wide band or narrow band. Due to mutual interference of light and dark modes, the Fano resonance of asymmetric line is produced. The simulated spectrum plotted with black solid lines is shown in Fig. 1(b), which explains that three transmission peaks with transmittances of $\sim 0.4$, $\sim 0.6$, and $\sim 0.2$ are generated at $510 \mathrm{~nm}, 610 \mathrm{~nm}$, and $890 \mathrm{~nm}$, respectively. Three steep dips, which have the lowest transmittances of $\sim 0$ at $520 \mathrm{~nm}$, $620 \mathrm{~nm}$, and $900 \mathrm{~nm}$, occur at the right sides of the transmission peaks, respectively. Moreover, there is a Lorentz peak at $1280 \mathrm{~nm}$ owing to FP resonance. All the simulations assume smooth metal surface. Although the sidewall roughness of the fabricated metal slot is quite large, this problem can be solved. Alimardani et al. [32] fabricated MIM tunneling diodes on various high and low power functional metals with different root mean square roughnesses by using the high-quality atom layer of $\mathrm{Al}_{2} \mathrm{O}_{3}$ deposited as the insulating layer. It is found that the surface roughness of the electrode can control the current and voltage characteristics of the MIM diode and overcome the influence of the metal working function. After extensive research by the authors, they believed that the roughness of the bottom electrode to be less than $20 \%$ of the thickness of the insulator in order to achieve nonroughness dominated electrical behavior.

The factors that affect the performance of refractive index sensing are as follows: firstly, the performance of refractive index sensing is closely related to the electromagnetic properties of the 
medium; secondly, the refractive index sensing performance is also related to wavelength, which is called dispersion phenomenon; thirdly, the gas refractive index sensing performance is also related to temperature and pressure; fourthly, it is also

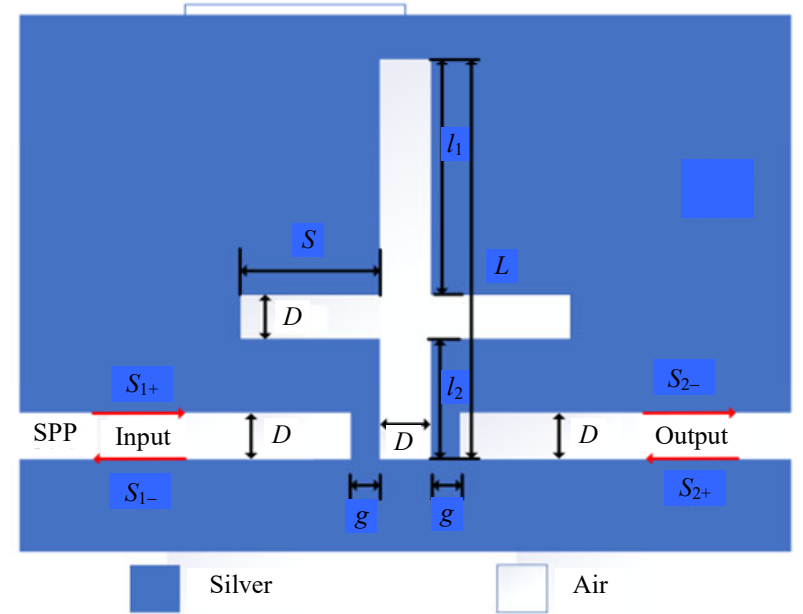

(a) closely related to the arrangement of ions, in which the isotropic optical materials will also affect the refractive index sensing performance. Thus, no matter how the refractive index changes, one can detect it through the shifts of the peaks.

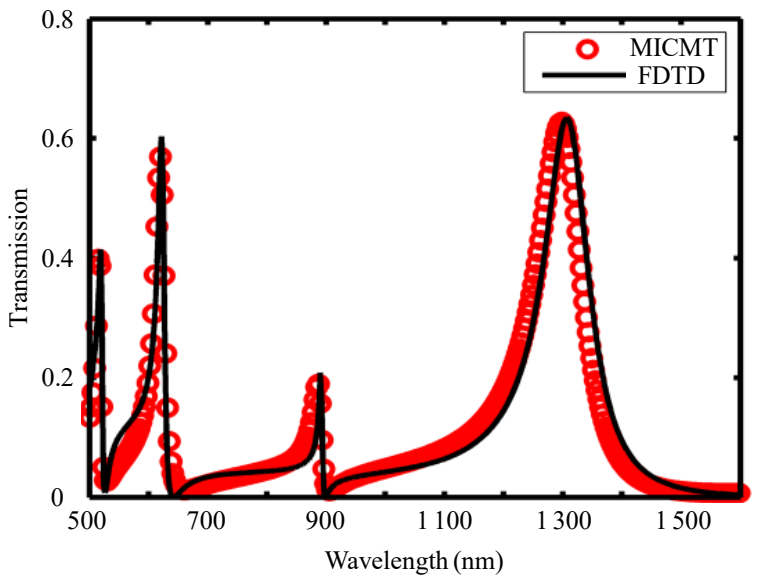

(b)

Fig. 1 Structural diagram and transmission spectra: (a) cross-shape cavity resonator structure and (b) transmission spectra of the basic structure based on FDTD (black solid line) and MICMT (red circle line) methods.

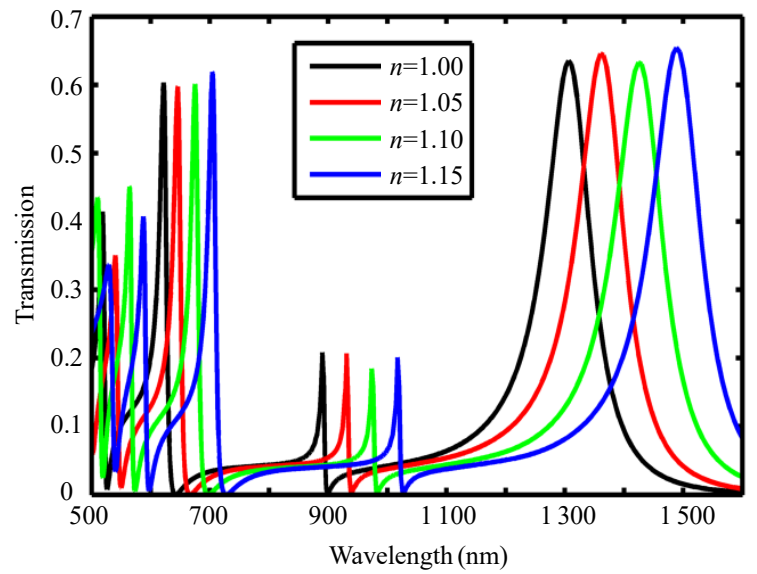

(a)

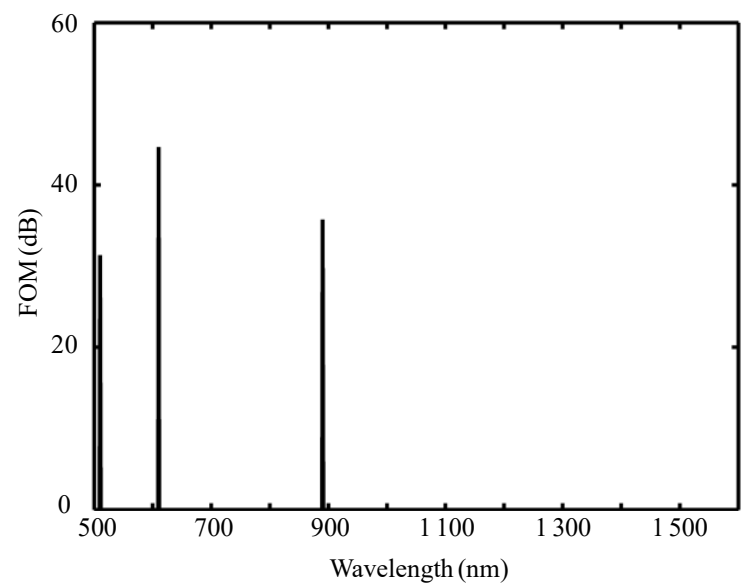

(b)

Fig. 2 Transmission spectra and sensor sensitivity under different refractive index conditions: (a) responses of the sensor for different refractive indices and (b) maximum FOM value of the proposed structure

The sensing performance of the proposed structure is demonstrated with responding to different refractive indices of the dielectric marked white in Fig. 1(a). The geometrical parameters of the structure are those given in the previous section. The refractive index has been varied from 1 to 1.15 with a step of 0.05 and the corresponding responses are shown in Fig. 2(a). It can be seen that the Fano-like response is preserved at all four resonances for each response. The sensitivity of a sensor (nm/RIU) is usually defined as the shift in the resonance wavelength per unit variation of the refractive index [33]. According to the responses, the calculated values are $400 \mathrm{~nm} / \mathrm{RIU}, \quad 700 \mathrm{~nm} / \mathrm{RIU}$, and $1000 \mathrm{~nm} / \mathrm{RIU}$ for the Fano peaks at $510 \mathrm{~nm}, 610 \mathrm{~nm}$, and $890 \mathrm{~nm}$, respectively. The sensitivity of $1000 \mathrm{~nm} / \mathrm{RIU}$ is higher than those of most similar MIM systems, meaning our system will be more 
sensitive to small variations of the surrounding. In a more detailed sensing application, once the refractive index of the medium changes with temperature, concentration or pressure, the displacement of the resonant peak can be measured to calculate the refractive index change. Therefore, the structure can be used for sensing applications in different refractive index environments, such as biological and medical applications, where the sensing material is a water-based solution. Moreover, in the aspect of refractive index sensing, the advantage of multiple Fano resonance is that it can be used not only in the refractive index sensitive region, but also in the fast/slow light region. The proposed structure can also be used to realize the integration and miniaturization of the photonic loop, which provides some theoretical reference for simultaneous detection and differential sensing of various samples to be tested.

In addition, figure-of-merit (FOM) is an important factor in evaluating sensor performance and can be expressed as [34]

$$
F O M=\max \left(\left|\frac{\mathrm{d} T(\lambda) / \mathrm{d} n(\lambda)}{T(\lambda)}\right|\right)
$$

where $T(\lambda)$ is the transmission, and $\mathrm{d} T(\lambda) / \mathrm{d} n(\lambda)$ is the transmittance change at fixed wavelength induced by a refractive index change. According to (1), it can be concluded that in order to obtain high FOM, ultra-low transmittance for the dip is preferred, and then the sharp increase caused by the change of index is obtained. Due to the super sharp asymmetric Fano line shape, the maximum FOM values at the Fano peaks of $510 \mathrm{~nm}, 610 \mathrm{~nm}$, and $890 \mathrm{~nm}$ are achieved as high as $\sim 31 \mathrm{~dB}, \sim 42 \mathrm{~dB}$, and $\sim 38 \mathrm{~dB}$, respectively, as exhibited in Fig. 2(b).

Secondly, as we all know, the coupled mode theory (CMT) $[35,36]$ is well-suited for analyzing single-mode coupling in plasmon-resonance systems because the coupling phase between the resonance mode and the waveguide can be ignored with an appropriate reference. However, when multiple mode coupling is involved, such as the structure proposed in Fig. 1(a), the coupling phase and modulus of each resonant mode are different, and they will affect transmission. Hence, the multimode interference coupled mode theory (MICMT) [35] is used to analyze the transmission spectrum for this proposed-structure, and gives the following four formulas:

$$
\begin{aligned}
& \frac{\mathrm{d} a_{n}}{\mathrm{dt}}=\left(-\mathrm{j} \omega_{n}-\frac{1}{\tau_{n i}}-\frac{1}{\tau_{n c 1}}-\frac{1}{\tau_{n c 2}}\right) a_{n}+ \\
& k_{n 1} S_{n, 1+}+k_{n 2} S_{n, 2+} \\
& S_{1-}=-S_{1+}+\sum_{n} k_{n 1}^{*} a_{n}, k_{n 1}=\sqrt{\frac{2}{\tau_{n c 1}}} \mathrm{e}^{\mathrm{j} \theta_{n 1}} \\
& S_{2-}=-S_{2+}+\sum_{n} k_{n 2}^{*} a_{n}, k_{n 2}=\sqrt{\frac{2}{\tau_{n c 2}}} \mathrm{e}^{\mathrm{j}\left(\theta_{n 2}-\varphi_{n}\right)} \\
& S_{n, 1+}=\gamma_{n 1} \mathrm{e}^{\mathrm{j} \varphi_{n 1}} S_{1+}, S_{n, 2+}=\gamma_{n 2} \mathrm{e}^{\mathrm{j} \varphi_{n 2}} S_{2+}
\end{aligned}
$$

where $a_{n}$ and $\omega_{n}$ are the normalized amplitude and resonant frequency of the $n$th resonant mode, respectively. $a_{n}$ is time dependent. $\tau_{n i}$ is the decay time of internal loss of $n$th resonant mode in resonator, whereas $\tau_{n c 1}$ and $\tau_{n c 2}$ are the decay time of the coupling between the resonator and waveguides. $\gamma_{n 1} \mathrm{e}^{\mathrm{j} \varphi_{n 1}}$ and $\gamma_{n 2} \mathrm{e}^{\mathrm{j} \varphi_{n 2}}$ stand for the normalized coefficients $\left(\gamma_{n 1}=\gamma_{n 1} \approx 1\right.$ in this paper). $\theta_{n 1}$ and $\theta_{n 2}$ are the coupling-phases of the $n$th resonant mode. $\varphi_{n}$ describes the phase difference between the output and input ports of the $n$-th resonant mode.

From Fig. 1(a), $S_{1,2+}$ is the normalized amplitude between the input and output MIM waveguides, respectively. Moreover, amplitude $S_{2+}$ can be set to zero, because the surface plasmon polaritons (SPPs) should be emitted from the left of MIM waveguide. Consequently, the amplitude transmission coefficient can be derived as the following formula:

$$
t=\frac{S_{2-}}{S_{2+}}=\sum_{n} \frac{\gamma_{n 1}\left|k_{n 1}\right|\left|k_{n 2}\right| \mathrm{e}^{\mathrm{j} \theta \phi_{n}}}{-\mathrm{j}\left(\omega-\omega_{n}\right)+\frac{1}{\tau_{n i}}+\frac{1}{\tau_{n c 1}}+\frac{1}{\tau_{n c 2}}}
$$

where $\phi_{n}=\varphi_{n 1}+\varphi_{n}+\theta_{n 1}-\theta_{n 2}$ is the coupling phase difference under the $n$th resonance mode; $\omega$ refers to the frequency range of the spectrum, which 
corresponds to $500 \mathrm{~nm}$ to $1600 \mathrm{~nm}$ in the diagram. Since the input and output waveguides are symmetrical with respect to the cross-shaped cavity and have a uniform width, the following conclusions can be drawn: $\tau_{n c 1}=\tau_{n c 2}=\tau_{n}$ and $\theta_{n 1}=\theta_{n 2}$, where $\tau_{n}$ is the number to replace $\tau_{n c 1}$ and $\tau_{n c 2}$. Therefore, the transmission $T$ of the structure can be expressed by the following formula:

$$
T=|t|^{2} \approx\left|\sum_{n} \frac{2 \mathrm{e}^{\mathrm{j} \theta \phi_{n}}}{-\mathrm{j}\left(\omega-\omega_{n}\right) \tau_{n}+2+\frac{\tau_{n}}{\tau_{n i}}}\right|^{2} .
$$

Finally, through the analysis of the FDTD method and the MICMT method mentioned above, the Fano-resonant peaks are further studied using the red circle line through the MICMT method shown in Fig. 1(b), which is in good agreement with the results obtained by the FDTD method.

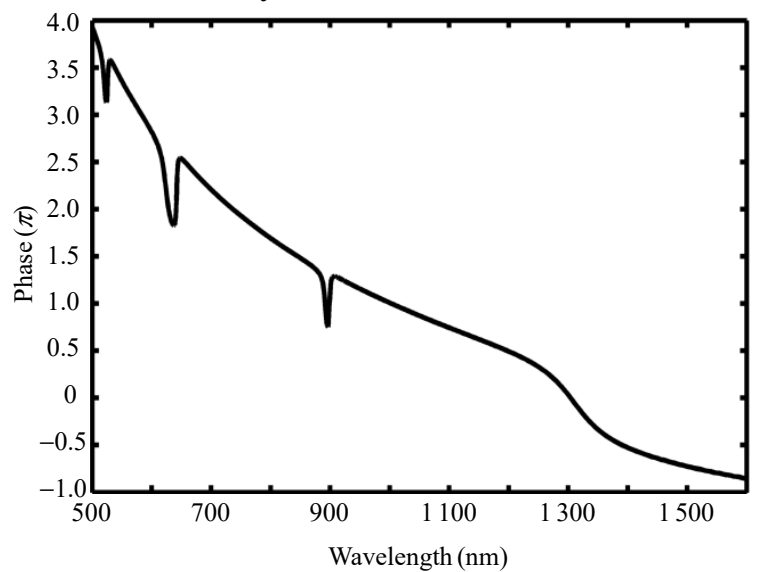

(a)

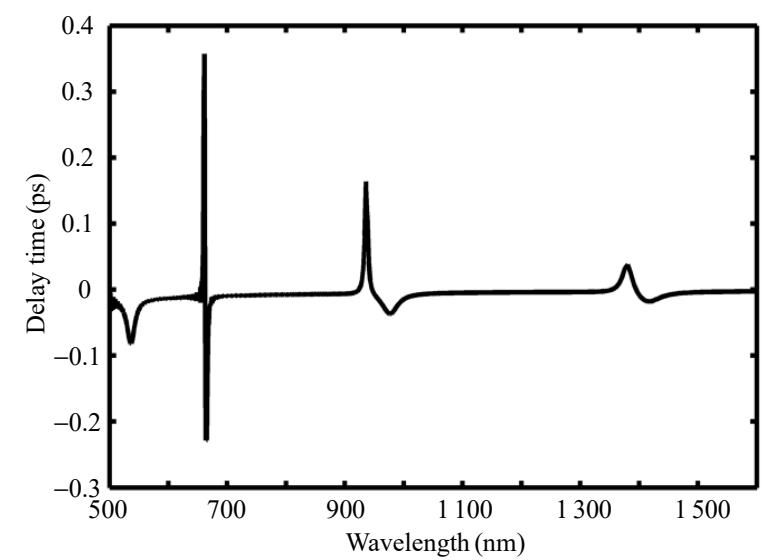

(b)

Fig. 3 Responses of phase and time delay: (a) phase responses and (b) delay time for the cross shaped rectangular cavity resonator structure, respectively.
Furthermore, in the application of fast or slow light technology, the phase response is a key factor, which can be attributed to the significant effect of resonance peaks and drops. It is important to note that the phase response is calculated using FDTD solution software from the electromagnetic field information obtained by the output port monitor. More details of the phase responses and the group delays for this structure are shown in Fig. 3. Especially, the phase changes between $500 \mathrm{~nm}$ and $1600 \mathrm{~nm}$ are shown in Fig.3(a), which shows phases are shifted from $3.1 \pi$ to $3.5 \pi$ at the wavelength range from $510 \mathrm{~nm}$ to $520 \mathrm{~nm}$, from $1.8 \pi$ to $2.5 \pi$ at the wavelength range from $620 \mathrm{~nm}$ to $650 \mathrm{~nm}$, and from $0.75 \pi$ to $1.3 \pi$ at the wavelength range from $890 \mathrm{~nm}$ to $900 \mathrm{~nm}$, respectively. Actually, the phase shifts are attributed to Fano resonances; consequently, the spectral regions of phase shifts are determined by the wavelength ranges of Fano windows. One can compare the wavelengths of Fano windows in Fig. 1(b) to that of phase shifts in Fig. 3(a), then it can be found that they occur at the same wavelength range. Specifically, phase continuity will be disrupted within the Fano window, but it changes linearly at other wavelengths. Furthermore, according to the relationship between the group delay $\tau$ and the phase shift $\theta$, we can obtain the delay time through the condition: $\tau(\lambda)=-\lambda^{2} \mathrm{~d} \theta / 2 \pi c \mathrm{~d} \lambda$. By calculating the phase response in Fig. 3(a), the variation of delay time with respect to wavelength is achieved in Fig. 3(b). It can be seen that group delays of $\sim-0.05 \mathrm{ps}, \sim-0.22 \mathrm{ps}$, and $\sim 0.03$ ps are obtained during three descent periods, respectively. In addition, we can know that group delays of $\sim 0.35 \mathrm{ps}$ and $\sim 0.18 \mathrm{ps}$ are obtained at the two peaks, respectively. Obviously, positive and negative delay time, which are preferred in slow/fast light area, are obtained at the wavelengths around each Fano peak and dip, respectively. Intuitively, additional positive delay time is pursued in the slow light communication system. Alternatively, the fast light system will employ the negative delay time characteristic. Generally speaking, in addition to the refractive index sensing, one can properly use the 
Fano window for the requirement of slow/fast light communication.

In Fig.4(a), the parameters are similar to that in Fig. 1(a), but a rectangular cavity is added at the left end. The width of the rectangular cavity is $D=50 \mathrm{~nm}$, the length of the rectangular cavity is $H=370 \mathrm{~nm}$, and the coupling distance is $g=10 \mathrm{~nm}$. Specifically, a large wide-band bright mode, which emerges in the left rectangular cavity, will interact with the dark mode in the cross-shape one. Due to the mutual mode interference, Fig. 4(b) shows the transmission spectrum of the structure, which generates five Fano resonant peaks. Obviously, the simulated spectrum plotted with black solid lines is shown in Fig.4(b), which explains that five transmission peaks with five transmittances of $\sim 0.30, \sim 0.41, \sim 0.38, \sim 0.24$,

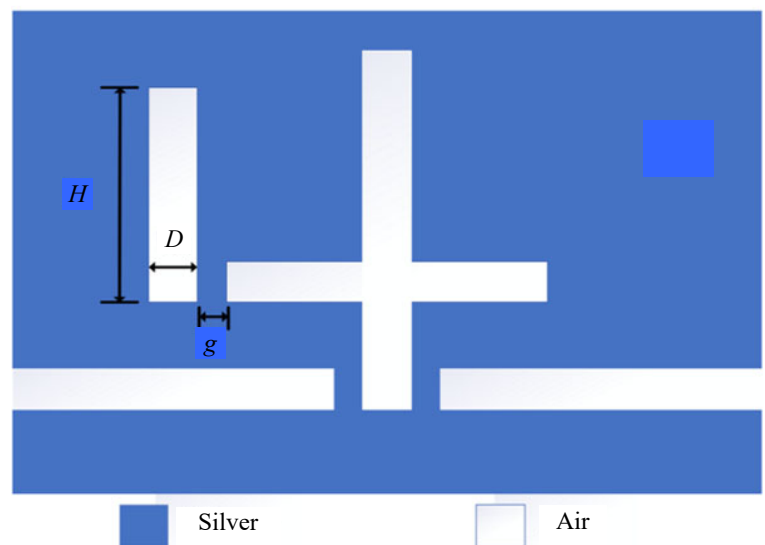

(a)

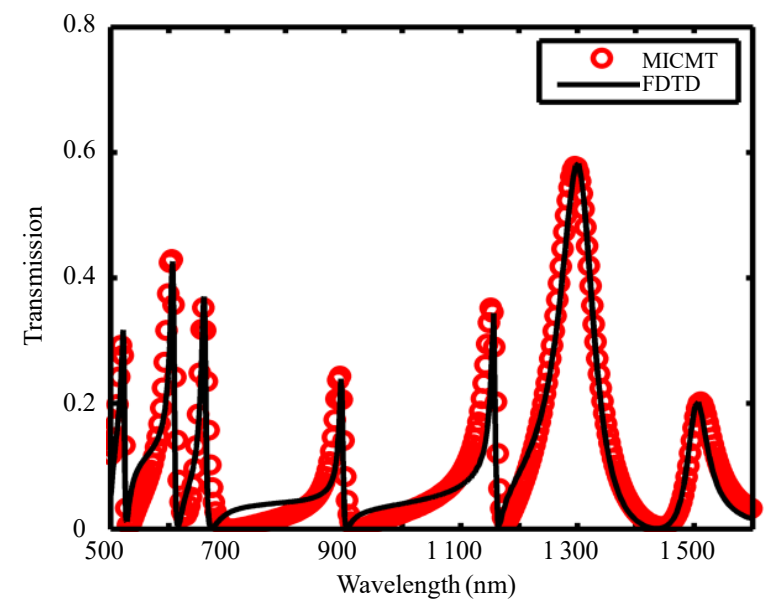

(b)

Fig. 4 Structure diagram and transmission spectra: (a) mutually perpendicular rectangular cavity and left end coupled rectangular cavity resonator structure and (b) transmission spectra of the basic structure based on FDTD (black solid line) and MICMT (red circle line) methods.

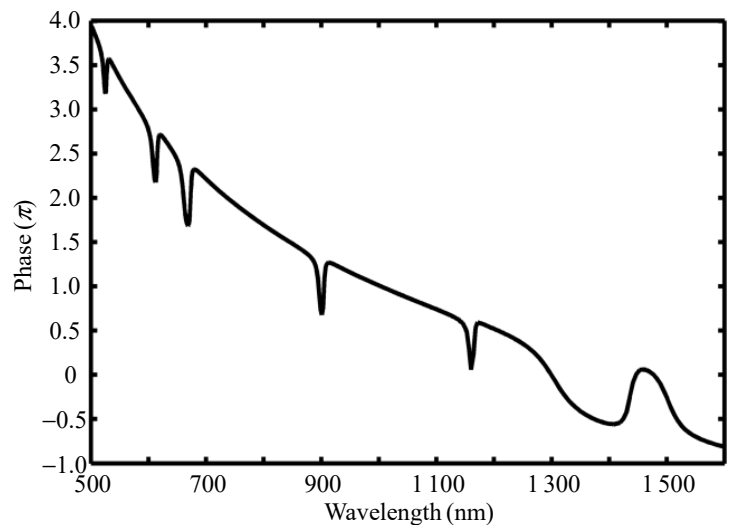

(a)

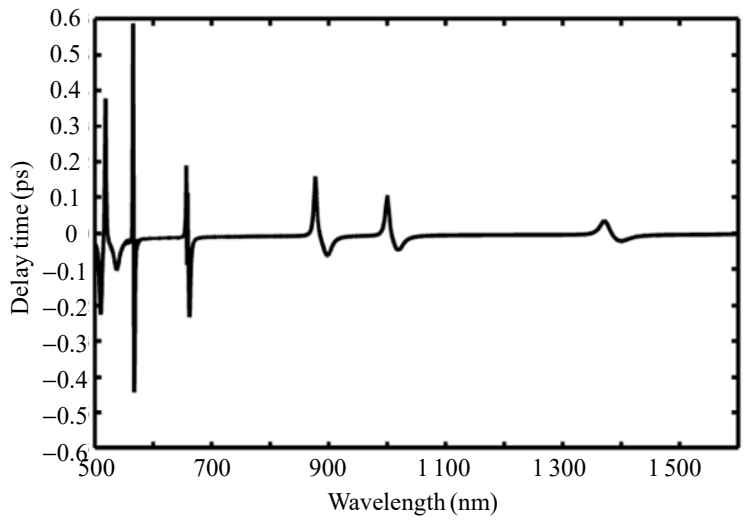

(b)

Fig. 5 Responses of phase and time delay: (a) phase responses and (b) delay time for the cross shaped rectangular cavity and left end coupled rectangular cavity resonator structure, respectively.

and $\sim 0.38$ are generated at $510 \mathrm{~nm}, 590 \mathrm{~nm}, 680 \mathrm{~nm}$, $890 \mathrm{~nm}$, and $1150 \mathrm{~nm}$, respectively. Five steep dips, which have the lowest transmittance of $\sim 0$ at $520 \mathrm{~nm}, 620 \mathrm{~nm}, 690 \mathrm{~nm}, 900 \mathrm{~nm}$, and $1160 \mathrm{~nm}$, are generated to the right of the transmission peaks, respectively. There are also two Lorentz peaks at $1290 \mathrm{~nm}$ and $1500 \mathrm{~nm}$. Similarly, through the analysis of FDTD method and MICMT method, the Fano-resonant peaks are further investigated by the MICMT method shown in Fig.4(b) using red circle line, which agrees well with the result of FDTD method.

Similar to the analysis above, phase shifts are achieved because of the Fano resonances in Fig.4(b), and negative group delay and positive group delay are obtained in the Fano dips and peaks in Fig. 5, respectively. Taking several phase changes and group delay in Fig. 5 as examples, phase changes occur at $510 \mathrm{~nm}$ and $620 \mathrm{~nm}$, respectively, 
corresponding to Fano resonance depression in Fig. 4(b). In the wavelength ranges of $510 \mathrm{~nm}$ to $520 \mathrm{~nm}$ and $620 \mathrm{~nm}$ to $650 \mathrm{~nm}$, the phase ranges from $3.1 \pi$ to $3.5 \pi$ and $2.2 \pi$ to $2.6 \pi$, respectively. Then, the group delay can be calculated, and the maximum delays at $510 \mathrm{~nm}$ and $620 \mathrm{~nm}$ are $-0.21 \mathrm{ps}$ and $0.19 \mathrm{ps}$, respectively. More detailed results are shown in Table 1. Consequently, the unique properties within these Fano resonant windows will play a significant role in the development of fast or slow light technologies in communication.

Table 1 Phase-shifts and group-delays corresponding to Fig. 4.

\begin{tabular}{|c|c|c|c|c|}
\hline & & Wavelength range & Wavelength range & Wavelength range \\
\hline \multirow{8}{*}{$\begin{array}{l}\text { Phase } \\
\text { shifts } \\
\text { and } \\
\text { group } \\
\text { delays }\end{array}$} & \multirow{4}{*}{$\begin{array}{c}\text { Positive } \\
\text { delay }\end{array}$} & $520 \mathrm{~nm}-530 \mathrm{~nm}$ & $560 \mathrm{~nm}-570 \mathrm{~nm}$ & $620 \mathrm{~nm}-650 \mathrm{~nm}$ \\
\hline & & $0,0.39 \mathrm{ps}$ & $0,0.59 \mathrm{ps}$ & $0.4 \pi, 0.19 \mathrm{ps}$ \\
\hline & & $890 \mathrm{~nm}-900 \mathrm{~nm}$ & $1000 \mathrm{~nm}-1010 \mathrm{~nm}$ & $1150 \mathrm{~nm}-1160 \mathrm{~nm}$ \\
\hline & & $0.55 \pi, 0.19 \mathrm{ps}$ & $0,0.18 \mathrm{ps}$ & $0.5 \pi,-0.03 \mathrm{ps}$ \\
\hline & \multirow{4}{*}{$\begin{array}{c}\text { Negative } \\
\text { delay }\end{array}$} & $510 \mathrm{~nm}-520 \mathrm{~nm}$ & $530 \mathrm{~nm}-550 \mathrm{~nm}$ & $570 \mathrm{~nm}-580 \mathrm{~nm}$ \\
\hline & & $0.4 \pi,-0.21 \mathrm{ps}$ & $0,-0.09 \mathrm{ps}$ & $0,-0.41 \mathrm{ps}$ \\
\hline & & $680 \mathrm{~nm}-690 \mathrm{~nm}$ & $910 \mathrm{~nm}-920 \mathrm{~nm}$ & $1020 \mathrm{~nm}-1030 \mathrm{~nm}$ \\
\hline & & $0.6 \pi,-0.22 \mathrm{ps}$ & $0,-0.07 \mathrm{ps}$ & $0,-0.05 \mathrm{ps}$ \\
\hline
\end{tabular}

Likewise, in Fig. 6(a), a rectangular cavity is added at the right end for obtaining more Fano channels, while other parameters are the same as that in Fig.4(a). The width of the rectangular cavity is $D=60 \mathrm{~nm}$, the length of the rectangular cavity is $G=370 \mathrm{~nm}$, and the coupling distance is $g=10 \mathrm{~nm}$. Similarly, due to the mode interferences from the right cavity and the cross-shape one, the simulated spectrum plotted with the black solid line is shown in Fig. 6(b), which explains that six transmission peaks with transmittances of $\sim 0.22, \sim 0.34, \sim 0.41$, $\sim 0.20, \sim 0.23$, and $\sim 0.58$ are generated at $510 \mathrm{~nm}$, $620 \mathrm{~nm}, 680 \mathrm{~nm}, 890 \mathrm{~nm}, 950 \mathrm{~nm}$, and $1150 \mathrm{~nm}$, respectively. Six steep dips, which have the lowest transmittances of $\sim 0$ at $520 \mathrm{~nm}, 650 \mathrm{~nm}, 690 \mathrm{~nm}$, $900 \mathrm{~nm}, 960 \mathrm{~nm}$, and $1160 \mathrm{~nm}$, are generated at the right of the transmission peaks, respectively, and there is a Lorentz peak achieved at $1400 \mathrm{~nm}$. Similarly, through the analysis of FDTD method and MICMT method, the Fano-resonant peaks are further investigated by the MICMT method shown in Fig. 6(b) using red circle line, which agrees well with the result of FDTD method.

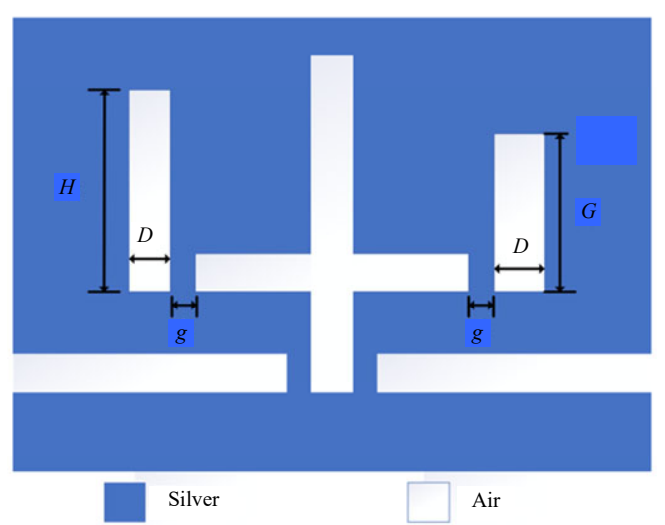

(a)

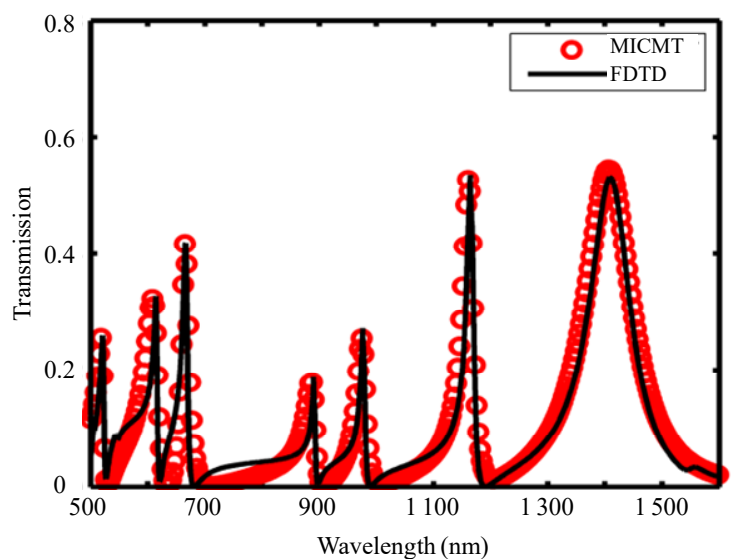

(b)

Fig. 6 Structure diagram and transmission spectra: (a) mutually perpendicular rectangular cavity and two ends coupled rectangular cavity resonator structure and (b) transmission spectra of the basic structure based on FDTD (black solid line) and MICMT (red circle line) methods.

Figures 7(a) and 7(b) show the phase responses and delay time corresponding to the structure in Fig. 6(a), respectively. Owing to Fano resonance, phase shifts are achieved within the Fano windows. In addition to the sensing applications, since there are multiple Fano peaks in the proposed structure, one can use the normal and abnormal dispersion performances in other areas, such as multi-channel optical communication system. Taking several phase changes and group delay in Fig. 7 as examples, phase changes occur at $510 \mathrm{~nm}$ and $620 \mathrm{~nm}$, respectively, corresponding to Fano-resonance depression in Fig. 6(b). In the wavelength ranges of $510 \mathrm{~nm}$ to $520 \mathrm{~nm}$ and $620 \mathrm{~nm}$ to $650 \mathrm{~nm}$, the phase ranges from $3.1 \pi$ to $3.5 \pi$ and $2.2 \pi$ to $2.6 \pi$, respectively. It also shows that obvious phase will occur within the Fano window, but the phase curve 


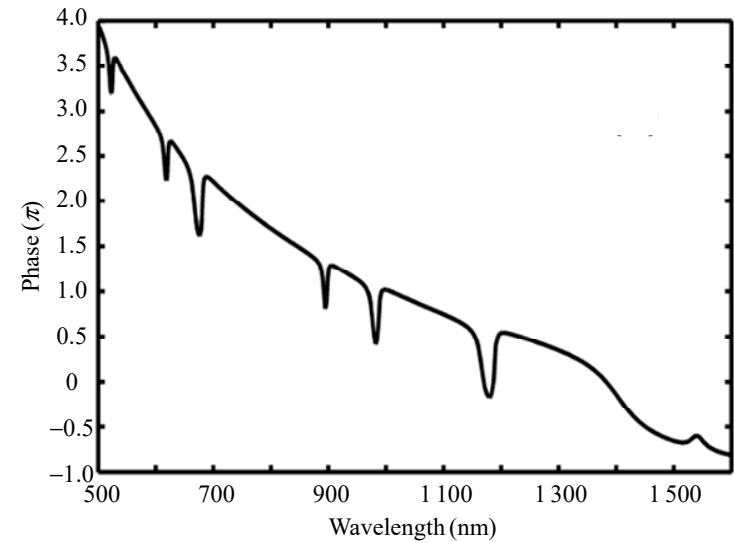

(a)

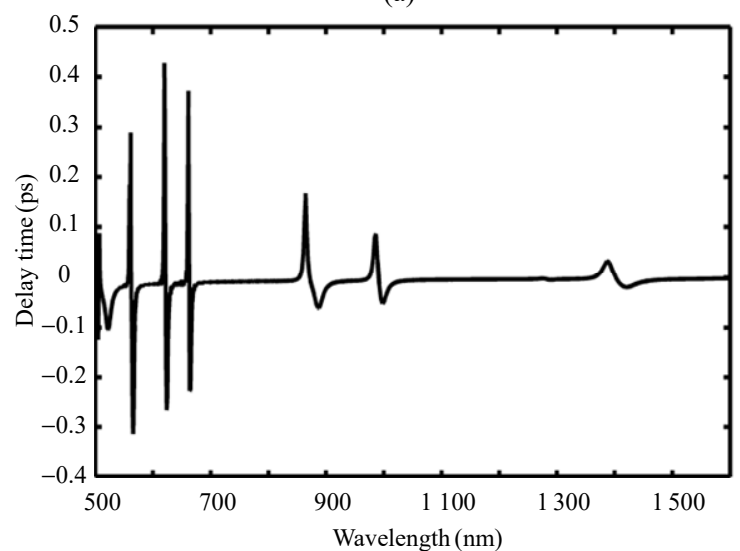

(b)

Fig. 7 Responses of phase and time delay: (a) phase responses and (b) delay time for the cross shaped rectangular cavity and two ends coupled rectangular cavity resonator structure, respectively.

is quite smooth at other wavelengths. According to the relationship between the group delay $\tau$ and the phase $\theta$, the group delay can be calculated, and the maximum delays at $510 \mathrm{~nm}$ and $620 \mathrm{~nm}$ are $-0.10 \mathrm{ps}$
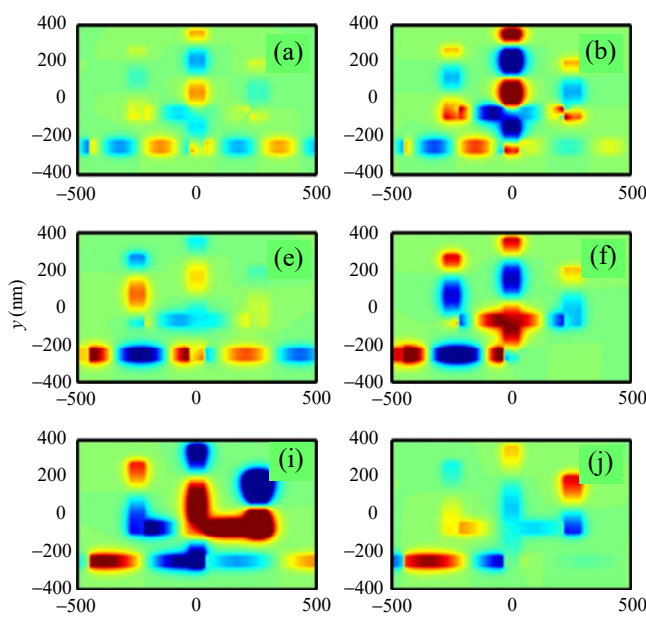

and $0.49 \mathrm{ps}$, respectively. More detailed results are shown in Table 2. Similarly, the unique properties within these Fano resonant windows will be preferred in the slow/fast light technologies in communication.

Table 2 Phase-shifts and group-delays corresponding to Fig. 6.

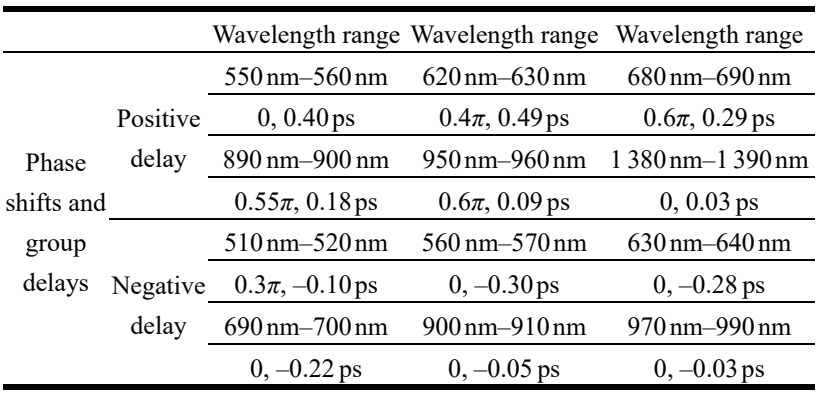

To understand the principle of the mode interaction that causes Fano resonance, Fig. 8 shows twelve magnetic field distributions of magnetic field distributions matching the structure in Fig.5(a). At the inclination wavelength, the magnetic field of the rectangular cavity at the right end is almost zero, as shown in Figs. 8(b), 8(d), 8(f), 8(h), and 8(l), respectively. Moreover, there is almost no SPP energy in the output MIM waveguide. The opposite distribution details can be found in Figs.8(a), 8(c), $8(\mathrm{e}), 8(\mathrm{~g}), 8(\mathrm{i})$, and $8(\mathrm{k})$, which illustrate the magnetic fields at the peak wavelengths. Besides, due to the strong interference between cross-shape cavity and the rectangular cavities on both sides, large magnetic field energy ratio causes multiple Fano resonances.
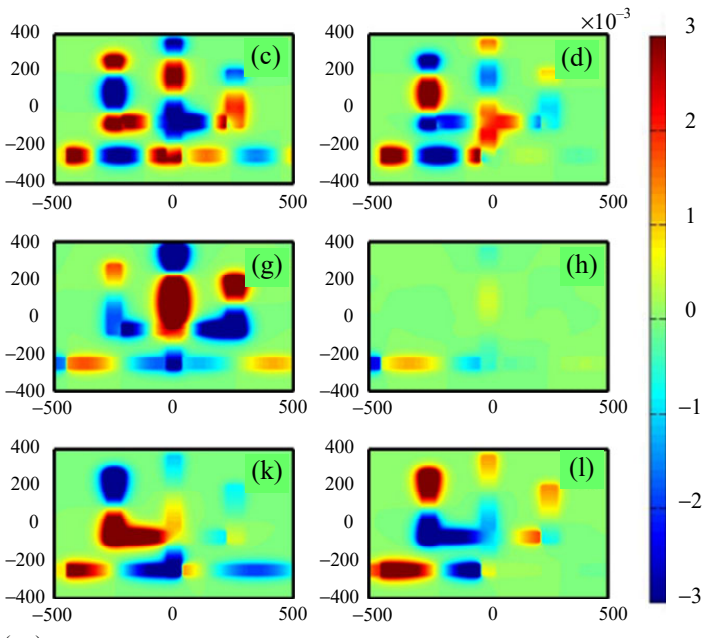

Fig. 8 Magnetic field distributions for the cross shaped rectangular cavity and two-end coupled rectangular cavity resonator: (a) peak at $518 \mathrm{~nm}$, (b) dip at $525 \mathrm{~nm}$, (c) peak at $614 \mathrm{~nm}$, (d) dip at $620 \mathrm{~nm}$, (e) peak at $665 \mathrm{~nm}$, (f) dip at $680 \mathrm{~nm}$, (g) peak at $890 \mathrm{~nm}$, (h) dip at $898 \mathrm{~nm}$, (i) peak at $975 \mathrm{~nm}$, (j) dip at $1015 \mathrm{~nm}$, (k) peak at $1164 \mathrm{~nm}$, and (l) dip at $1185 \mathrm{~nm}$. 


\section{Conclusions}

Transmission characteristics of SPPs-based MIM waveguides in the cross-shape rectangular cavity and two end-coupled rectangular cavity resonators have been proposed and investigated. The results show that all the cavities can effectively provide the bright or dark modes that interact with each other and up to six Fano resonant peaks have been achieved. In addition, the negative and positive group delays are also observed in Fano windows, and the proposed structure can not only be used in the refractive index sensing area, and also be used in the fast/slow light region. The performances of the proposed structure have been investigated by FDTD simulation and analyzed by MICMT. It can be considered that the proposed structure has broad application prospects in the field of on-chip optical sensing.

\section{Acknowledgment}

The authors would like to thank the editor and the reviewers for their valuable comments and suggestions, which help improve the quality of the paper.

Open Access This article is distributed under the terms of the Creative Commons Attribution 4.0 International License (http://creativecommons.org/licenses/by/4.0/), which permits unrestricted use, distribution, and reproduction in any medium, provided you give appropriate credit to the original author(s) and the source, provide a link to the Creative Commons license, and indicate if changes were made.

\section{References}

[1] U. Fano, "Effects of configuration interaction on intensities and phase shifts," Physical Review, 1961, 124(6): 1866-1878.

[2] B. Luk'Yanchuk, N. I. Zheludev, S. A. Maier, N. J. Halas, P. Nordlander, H. Giessen, et al., "The Fano resonance in plasmonic nanostructures and metamaterials," Nature Materials, 2010, 9(9): 707-715.

[3] M. F. Limonov, M. V. Rybin, A. N. Poddubny, and Y. S. Kivshar, "Fano resonances in photonics," Nature Photonics, 2017, 11(9): 543-554.
[4] G. T. Cao, H. J. Li, Y. Deng, S. Zhan, Z. He, and B. $\mathrm{Li}$, "Plasmon-induced transparency in a single multimode stub resonator," Optics Express, 2014, 22(21): 25215-25223.

[5] Y. K. Gong, X. M. Liu, and L. R. Wang, "Highchannel-count plasmonic filter with the metal-insulator-metal Fibonacci-sequence gratings," Optics Letters, 2010, 35(3): 285-287.

[6] X. Luo, X. H. Zou, X. F. Li, Z. Zhou, W. Pan, L. Yan, et al., "High-uniformity multichannel plasmonic filter using linearly lengthened insulators in metal-insulator-metal waveguide," Optics Letters, 2013, 38(9): 1585-1587.

[7] R. E. Holmlin, R. Haag, M. L. Chabinyc, R. F. Ismagilov, A. E. Cohen, A. Terfort, et al., "Electron transport through thin organic films in metal-insulator-metal junctions based on self-assembled monolayers," Journal of the American Chemical Society, 2001, 123(21): 5075-5085.

[8] F. Galvez, J. Del Valle, A. Gomez, M. R. Osorio, D. Granados, D. P. de Lara, et al., "Plasmonic nanodevice with magnetic funcionalities: fabrication and characterization," Optical Materials Express, 2016, 6(10): 3086-3096.

[9] S. P. Zhan, H. J. Li, G. T. Cao, Z. He, B. Li, and H. Yang, "Slow light based on plasmon-induced transparency in dual-ring resonator-coupled MDM waveguide system," Journal of Physics D Applied Physics, 2014, 47(20): 205101.

[10] Z. Chen, J. J. Chen, L. Yu, and J. Xiao, "Sharp trapped resonances by exciting the anti-symmetric waveguide mode in a metal-insulator-metal resonator," Plasmonics, 2015, 10(1): 131-137.

[11] S. B. Yan, L. Luo, C. Y. Xue, and Z. D. Zhang, "A refractive index sensor based on a metal-insulator-metal waveguide-coupled ring resonator," Sensors, 2015, 15(11): 29183-29191.

[12] W. L. Barnes, A. Dereux, and T. W. Ebbesen, "Surface plasmon subwavelength optics," Nature, 2003, 424(6950): 824-830.

[13] J. A. Dionne, L. A. Sweatlock, H. A. Atwater, and A. Polman, "Plasmon slot waveguides: Towards chip-scale propagation with subwavelength-scale localization," Physical Review B, Condensed Matter And Materials Physics, 2006, 73(3): 035407.

[14] Y. D. Jin, N. Friedman, M. Sheves, and D. Cahen, "Bacteriorhodopsin-monolayer-based planar metal-insulator-metal junctions via biomimetic vesicle fusion: preparation, characterization, and bio-optoelectronic characteristics," Advanced Functional Materials, 2007, 17(8): 1417-1428.

[15] Q. Zhu and H. K. Tsang, "High coupling efficiency silicon waveguide to metal-insulator-metal waveguide mode converter," Journal of Lightwave Technology: A Joint IEEE/OSA Publication, 2016, 34(10): 2467-2472. 
[16] K. H. Wen, Y. H. Hu, L. Chen, J. Zhou, L. Lei, and Z. Guo, "Fano resonance with ultra-high figure of merits based on plasmonic metal-insulator-metal waveguide," Plasmonics, 2015, 10(1): 27-32.

[17] G. C. Wang, A. Shen, C. Y. Zhao, L. Yang, T. Dai, Y. Wang, et al., "Fano-resonance-based ultra-high-resolution ratio-metric wavelength monitor on silicon," Optics Letters, 2016, 41(3): 544-547.

[18] H. Q. Liang, B. Liu, J. F. Hu, and X. D. He, "High efficiency all-optical plasmonic diode based on a nonlinear side-coupled waveguide-cavity structure with broken symmetry," Optics Communications, 2018, 414: 98-101.

[19] B. Sun, L. X. Zhao, C. Wang, X. Yi, Z. Liu, G. Wang, et al., "Tunable Fano resonance in E-shape plasmonic nanocavities," Journal of Physical Chemistry C, 2014, 118(43): 25124-25131.

[20] Z. Chen, L. Yu, L. L. Wang, Y. Zhao, and J. Xiao, "Sharp asymmetric line shapes in a plasmonic waveguide system and its application in nanosensor," Journal of Lightwave Technology, 2015, 33(15): 3250-3253.

[21] V. A. Fedotov, M. Rose, S. L. Prosvirnin, N. Papasimakis, and N. I. Zheludev, "Sharp trapped-mode resonances in planar metamaterials with a broken structural symmetry," Physical Review Letters, 2007, 99(14): 147401.

[22] J. J. Chen, Z. Li, J. Li, and Q. Gong, "Compact and high-resolution plasmonic wavelength demultiplexers based on Fano interference," Optics Express, 2011, 19(10): 9976-9985.

[23] X. J. Piao, S. Yu, S. Koo, K. Lee, and N. Park, "Fano-type spectral asymmetry and its control for plasmonic metal- insulator-metal stub structures," Optics Express, 2011, 19(11): 10907-10912.

[24] Z. Chen and L. Yu, "Multiple Fano resonances based on different waveguide modes in a symmetry breaking plasmonic system," IEEE Photonics Journal, 2014, 6(6): 1-8.

[25] S. B. Yan, M. Zhang, X. F. Zhao, Y. Zhang, J. Wang, and W. Jin, "Refractive index sensor based on a metal-insulator-metal waveguide coupled with a symmetric structure," Sensors, 2017, 17(12): 2879.

[26] R. Zafar, S. Nawaz, G. Singh, A. d'Alessandro, and
M. Salim, "Plasmonics-based refractive index sensor for detection of hemoglobin concentration," IEEE Sensors Journal, 2018, 18(11): 4372-4377.

[27] Z. Li, W. W. Liu, Z. C. Hua, H. Cheng, S. Chen, and J. Tian, "Fano-resonance-based mode-matching hybrid metasurface for enhanced second-harmonic generation," Optics Letters, 2017, 42(16): 3117-3120.

[28] P. B. Johnson and R. W. Christy, “Optical constants of the noble metals," Physical Review B, 1972, 6(12): 4370-4379.

[29] D. Wei, "The study on the structure, plasmonic resonance modes and SERS properties of metallic nanoparticle arrays fabricated by nanosphere lithography," Ph.D. dissertation, Nanjing University, 2017.

[30] H. T. Miyazaki and Y. Kurokawa, "Squeezing visible light waves into a 3-nm-thick and 55-nm-long plasmon cavity," Physical Review Letters, 2006, 96(9): 097401.

[31] K. H. Wen, Y. H. Hu, L. Chen, J. Zhou, L. Lei, and Z. Meng, "Single/dual Fano resonance based on plasmonic metal-dielectric-metal waveguide," Plasmonics, 2016, 11(1): 315-321.

[32] N. Alimardani, E. C. William, J. F. Wager, and J. F. C. Jr, "Impact of electrode roughness on metal-insulator-metal tunnel diodes with atomic layer deposited $\mathrm{Al}_{2} \mathrm{O}_{3}$ tunnel barriers," Journal of Vacuum Science \& Technology A Vacuum Surfaces \& Films, 2012, 30(1): 01A113.

[33] Y. Y. Zhang, S. L. Li, Z. Chen, P. Jiang, R. Jiao, Y. Zhang, et al., "Ultra-high sensitivity plasmonic nanosensor based on multiple Fano resonance in the MDM side-coupled cavities," Plasmonics, 2017, 12(4): 1099-1105..

[34] H. A. Haus and W. Huang, "Coupled-mode theory," Proceedings of the IEEE, 2002, 79(10): 1505-1518.

[35] Q. Li, T. Wang, Y. K. Su, M. Yan, and M. Qiu, "Coupled mode theory analysis of mode-splitting in coupled cavity system," Optics Express, 2010, 18(8): 8367-8382.

[36] S. L. Li, Y. L. Wang, R. Z. Jiao, L. Wang, G. Duan, and $\mathrm{L}$. Yu, "Fano resonances based on multimode and degenerate mode interference in plasmonic resonator system," Optics Express, 2017, 25(4): 3525-3533. 